$$
\text { "Zeitler_Camp" — 2009/5/28 — 21:24 — page } 1 \text { - \#1 }
$$

\title{
Reflecting upon reflections
}

\author{
H. ZeitleR and D. CAMP
}

Abstract. This paper considers many applications of reflections in geometry. It begins with a few motivational problems for the classroom and goes on to consider the formal application to cases involving reflections across one line, two lines and three lines. It wraps up with a summary of results for reflections in higher orders.

All this stuff was treated in German and American schools too - so the paper is a typical example of German-American didactics.

Thinking is one of the greatest pleasure of mankind. Galileo Galilei

Key words and phrases: reflection.

ZDM Subject Classification: G10.

Most students and teachers of secondary school mathematics are acquainted with reflections across a line, because this is often discussed in elementary analytic geometry. However, many of the beautiful subtleties of reflections are never seen at this level. Here we will consider a lot of questions that naturally pop up when studying reflections, though we will not be able to answer them all. The hope is that students will be motivated to investigate the world of reflections in more depth.

Let us begin with same teasers to whet mental appetite:

1. Suppose that John, who is 1.75 meters tall, stands in front of a mirror, as shown in Figure 1. What is the smallest height of a vertical mirror that will allow John to see his full image? How does the result change with respect to John's distance from the mirror?

2. Suppose the angle between two adjacent mirrors is $\alpha$. A ray of light meets one of the mirrors with an angle of incidence $\varphi$, where $\varphi<\alpha$. Calculate the

Copyright (c) 2009 by University of Debrecen 


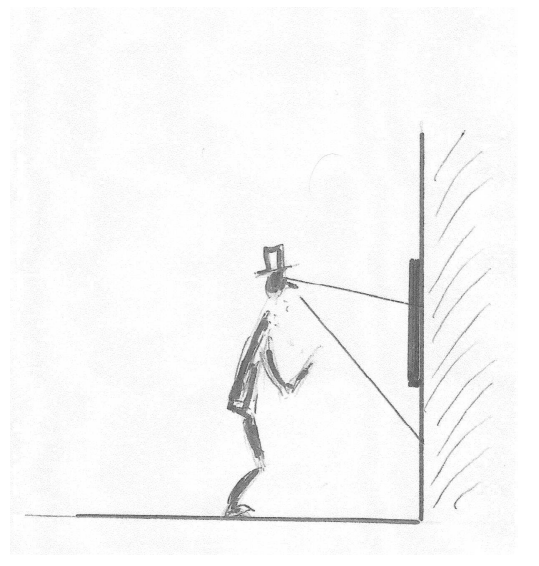

Figure 1. John in the mirror

angle $\beta$ shown in Figure 2. (Hint: Use two reflections and the fact that the angle of incidence is equal to the angle of reflection.) How does the situation change if $\varphi \geq \alpha$ ?

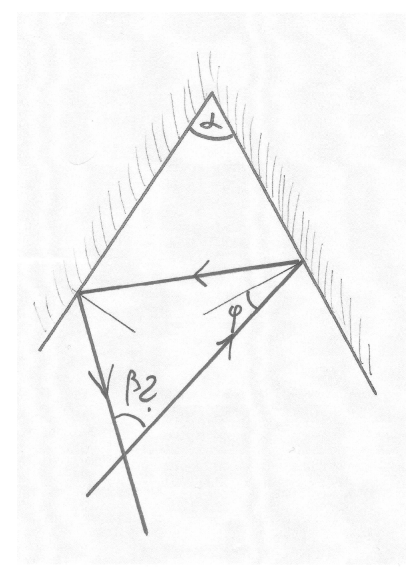

Figure 2. A ray of light within an angular mirror

3. On a billiard table, a ball starts at point $S$. If it is to meet the stationary ball situated at point $K$, as shown in Figure 3, after hitting only one cushion, what is its path? Suppose that we required it to two, or even three cushions 


$$
\text { "Zeitler_Camp" — 2009/5/28 - 21:24 — page } 3 \text { - \#3 }
$$

first. How does this change the path? (Hint: Figure 4 shows the billiard table with a mirror behind it... reflect on that!)

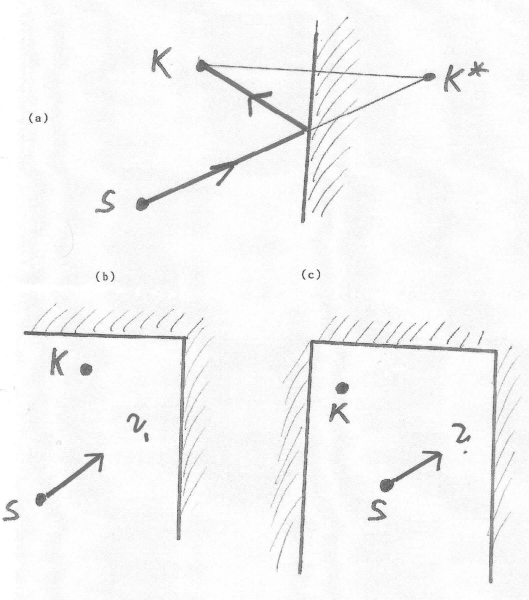

Figure 3. Playing billiards

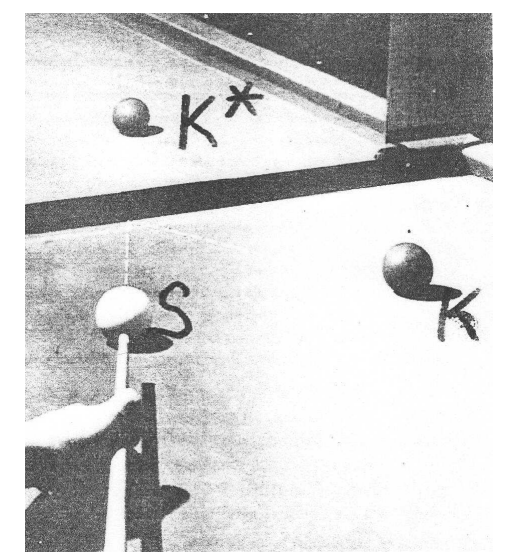

Figure 4. The billiard table

4. Suppose we have an acute triangle $A B C$ as shown in Figure 5. Let the edges $\overline{A B}$ and $\overline{B C}$ be mirrored. Suppose that a ray of light emanates from a point $X$ on $\overline{A B}$. Is it possible that this ray will find its way back to $X$ after two 


$$
\text { "Zeitler_Camp" — 2009/5/28 - 21:24 — page } 4 \text { - \#4 }
$$

reflections? Suppose that $A B C$ was an equilateral triangle and all of the edges were mirrored and the ray of light emanated from a point $X$ inside the triangle. Is there such a point where the ray will come back through $X$ after reflection through each of the three edges?

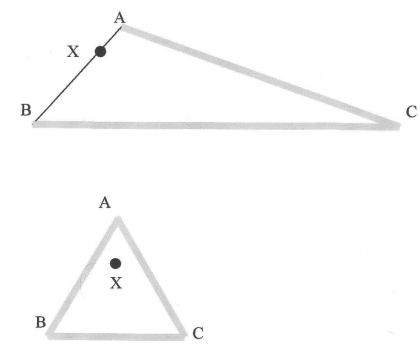

Figure 5. Triangular reflections

5. Nina is standing between two mirrors that meet at a $90^{\circ}$ angle in Figure 6 . She claims that she can see three copies of herself. How so? She also claims that if she raises her right arm, then two pictures move on the left but only one on the right. What does she mean? What would happen if the angle was an angle less than $90^{\circ}$ ?

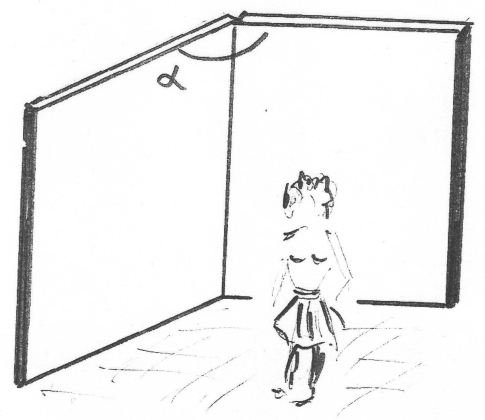

Figure 6. Nina in the double mirror 


$$
\text { "Zeitler_Camp" — 2009/5/28 - 21:24 — page } 5 \text { - \#5 }
$$

Let's stop now and consider some formal elements of reflection geometry. To do so, we will have to agree on how to indicate composition of reflections. We will indicate this "product" with a small circle "o". For example $\sigma_{1} \circ \sigma_{2} \circ \sigma_{3}$ applies the three reflections in numerical order. Here are some results:

Given two lines, $r$ and $s$, with corresponding reflections, $\sigma_{r}$ and $\sigma_{s}$ what happens if we apply them one after another $\sigma_{r} \circ \sigma_{s}$ ? There are two cases:

1. $r \| s$

- If $r=s$, we have the identity and nothing happens.

- If $r$ and $s$ have a distance $d>0$, then we get a translation $\tau$ with a magnitude of $2 d$, as shown in Figure 7. $X X^{\prime}=2 x+2(d-x)=2 d$, and $\overline{X X^{\prime}}$ orthogonal to $r$ and $s$. Thus $\tau=\sigma_{r} \circ \sigma_{s}$.

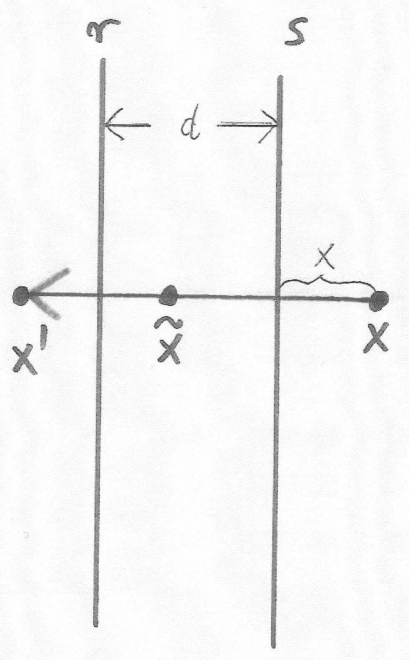

Figure \%. Translation $\tau$

2. $r \nVdash s$

- If $\alpha \neq 90^{\circ}$, as we can see in Figure 8, $m \angle X S X^{\prime}=2 \varphi+2(\alpha-\varphi)=2 \alpha$. This is simply a rotation, $\rho$, with center $S$. So $\rho=\sigma_{r} \circ \sigma_{s}$.

- If $\alpha=90^{\circ}$, we get the special case of the half-turn shown in Figure 9, a so called "point reflection" in $S$. We symbolize this $\tau=\sigma_{r} \circ \sigma_{s}$. 


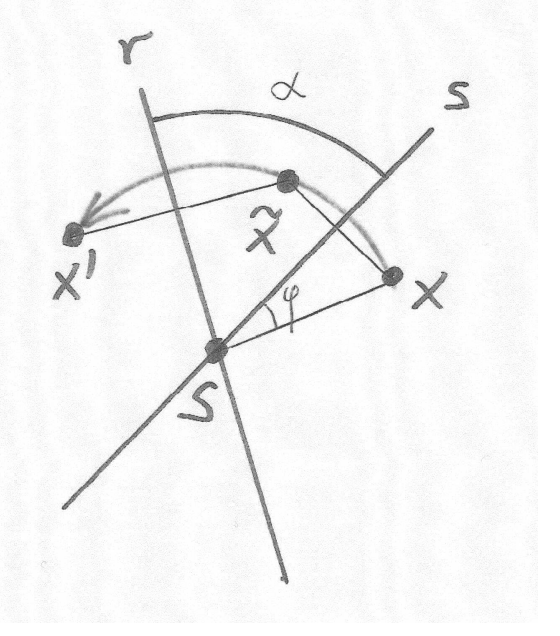

Figure 8. Rotation $\rho$

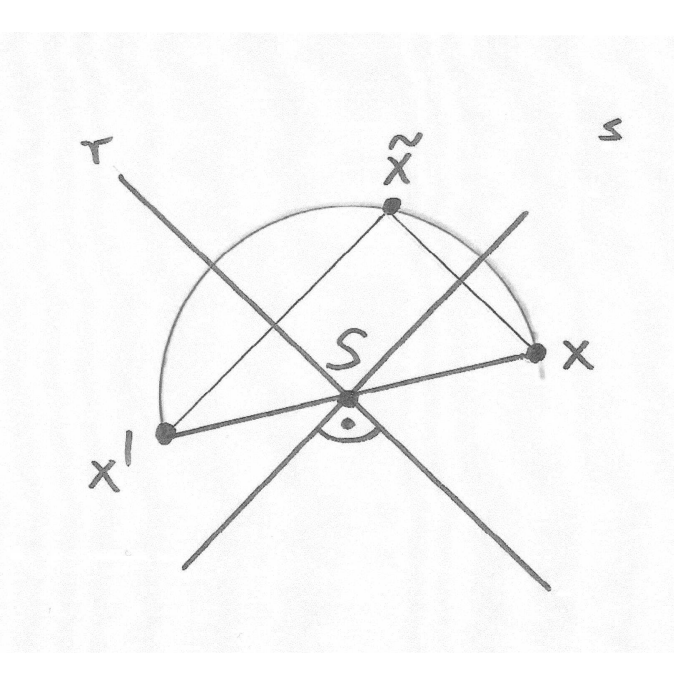

Figure 9. The special case of a point reflection $\tau$

In summary, we get a theorem about composition: the application of two reflections yields one of three fundamental mappings: the identity (id), a rotation $(\rho)$, or a translation $(\tau)$. But what about the converse? Can every rotation and every translation be decomposed into two reflections? The answer is yes. 
First, let's look at the case of the translation, $\tau$, illustrated in Figure 7 . Let $\tau$ be given by two points $X$ and $X^{\prime}$ with a distance of $2 d$ between them. If we choose an arbitrary line, $s$, orthogonal to the line $\overleftrightarrow{X X^{\prime}}$ and reflect $X$ through $s$, we get a point, $\widetilde{X}$. The perpendicular bisector of the segment $\overline{X^{\prime}} \widetilde{X}$ gives the line $r$. So we have $\tau=\sigma_{s} \circ \sigma_{r}$, where the distance between $r$ and $s$ is $d$. The proof for rotations runs analogously. (Here we should add one word of caution: with each one of the mappings, we are not simply operating on a single point or a special figure, we are affecting the whole plane.)

Now let us "up the ante" and look at the cases for 3 -fold reflections. We will start with lines $r, s, t$ and the corresponding reflections $\sigma_{r}, \sigma_{s}, \sigma_{t}$. Again we have to consider cases:

1. $r, s$ and $t$ are all three parallel.

- The cases where $r=s=t$ and $r=s \neq t$ are trivial, we obtain a reflection in one of the given lines.

- In general, the composition yields a reflection in a fourth line, $x$, parallel to $r$. As illustrated in Figure 10, we know $\tau=\sigma_{r} \circ \sigma_{s}$, but any translation can be decomposed into two reflections where one line is given, so we also have $\tau=\sigma_{x} \circ \sigma_{t}$. Thus, it follows that $\left(\sigma_{r} \circ \sigma_{s}\right) \circ \sigma_{t}=\tau \circ \sigma_{t}=$ $\sigma_{x} \circ \sigma_{t} \circ \sigma_{t}=\sigma_{x}$ where the line $x$ is the perpendicular bisector of $\overline{X X^{\prime}}$.

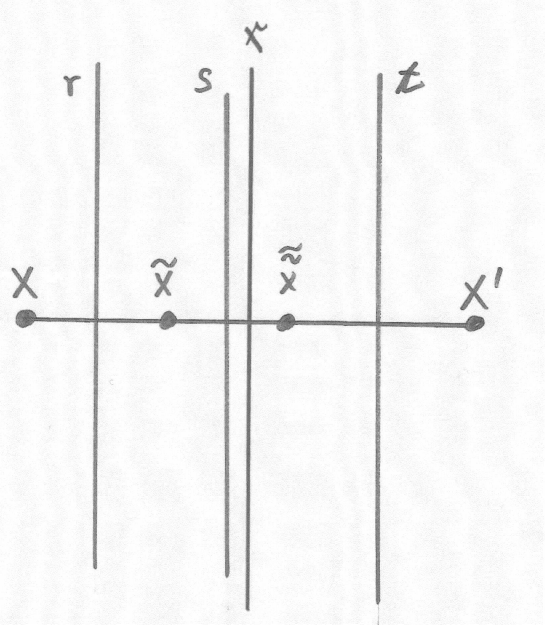

Figure 10. Three reflections 
2. $r, s$ and $t$ are not all parallel.

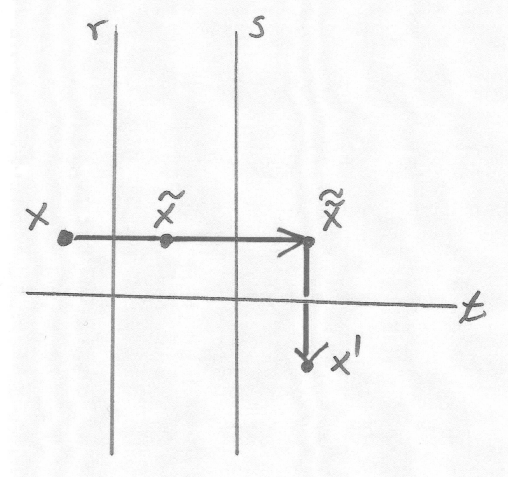

Figure 11. Glide reflection $\tau \sigma$

- There is a special case: $r \| s, r \neq s$ and $t \perp s$. Figure 11 illustrates this "glide reflection". This is a translation composed with a line reflection. We will symbolize this as $\tau \sigma$. Besides the mappings of id, $\tau$ and $\rho$, there exists another fundamental mapping, $\tau \sigma$. Figure 12 shows two real life examples-tracks in the show and paddling.

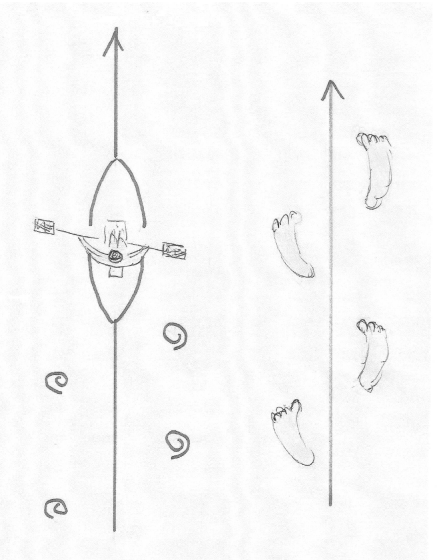

Figure 12. Tracks in the snow and paddling 


$$
\text { "Zeitler_Camp" — 2009/5/28 - 21:24 — page } 9 \text { - \#9 }
$$

- $r, s$ and $t$ intersect in a single point $S$. This composition yields a reflection in a line $x$ through $S$. As illustrated in Figure 13, we know $\rho=\sigma_{r} \circ \sigma_{s}$ and $\rho=\sigma_{x} \circ \sigma_{t}$ analogous to the parallel line case. Now it follows that $\left(\sigma_{r} \circ \sigma_{s}\right) \circ \sigma_{t}=\rho \circ \sigma_{t}=\sigma_{x} \circ \sigma_{t} \circ \sigma_{t}=\sigma_{x}$, as before. The line $x$ is the perpendicular bisector of the angle $X S X^{\prime}$.

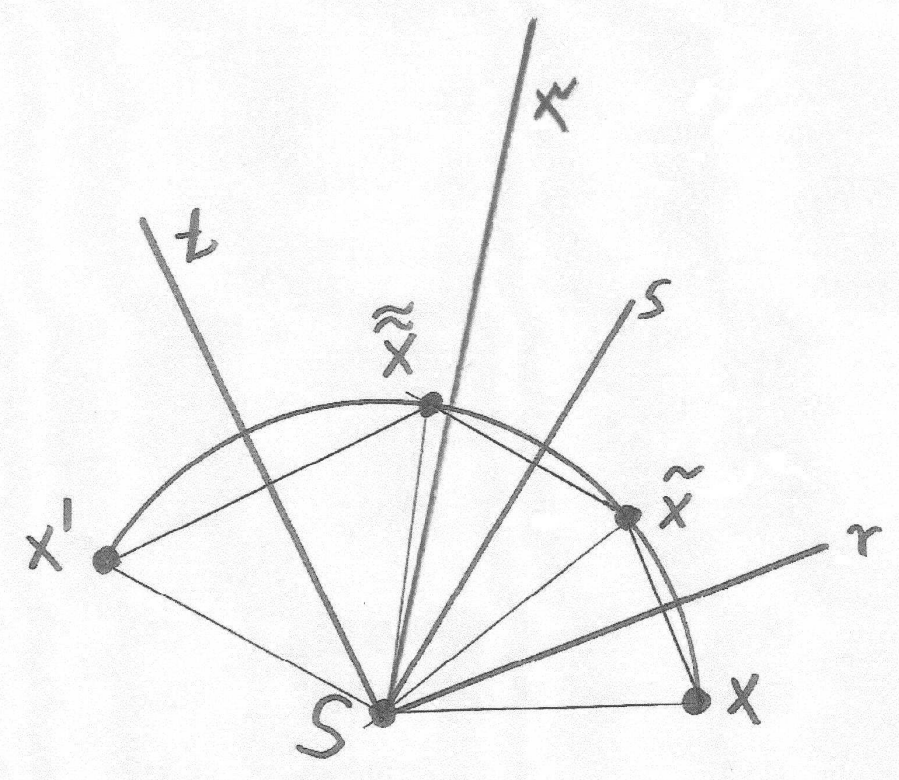

Figure 13. Concurrent lines

- $r, s$ and $t$ are pairwise not parallel and they do not intersect in one point. In other words, they form a triangle. In this complicated case we again get a glide reflection, by using the decomposition trick for rotations two times. The procedure is illustrated in the pictures presented in Figure 14: Start with a triangle as shown (a). (b) Let $s$ and $A$ be fixed, rotate $r$ and $t$ around $A$ until as $r \perp s$. The new elements are denoted by $\tilde{r}, \tilde{t}$, $\widetilde{B}$ and $\widetilde{C}$. (c) Now fix $\tilde{t}$ and $\widetilde{B}$ and rotate $\tilde{r}$ and $s$ around $\widetilde{B}$ until $\tilde{r} \perp \tilde{t}$, calling the new elements $\widetilde{A}$ and $\tilde{\tilde{r}}$. 
a)

b)

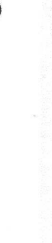

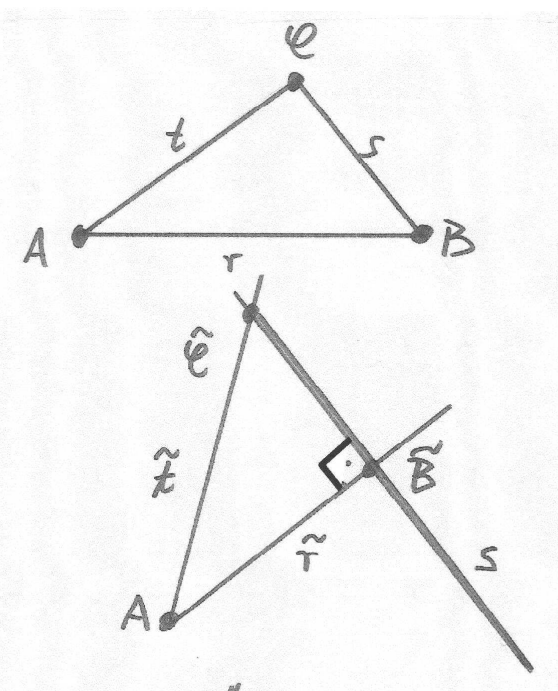

c)

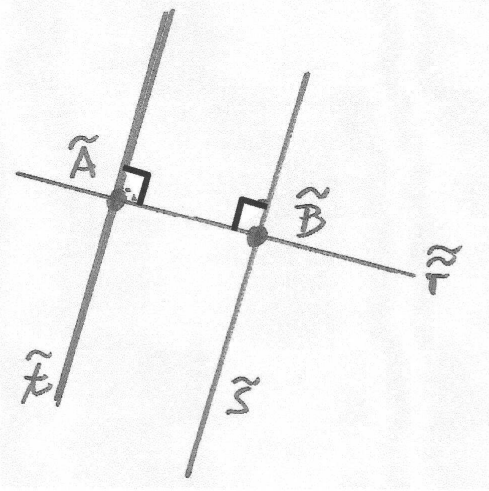

Figure 14. The complicated case

- There is a special case: $r \| s, r \neq s$ and $t \not \perp s$. This composition again yields a glide reflection, $\sigma \tau$. The proof is similar to the previous case and is illustrated in Figure 15. We start with parallel lines $r$ and $s$ cut by a nonorthogonal transversal as shown (a). (b) Let $s$ and $A$ be fixed again, and rotate $r$ and $t$ around $A$ until $t \perp s$. The new elements are denoted by $\tilde{r}, \tilde{t}, \widetilde{B}$ and $\widetilde{C}$. (c) Now fix $\tilde{r}$ and $\widetilde{B}$ and rotate $\tilde{t}$ and $s$ around $\widetilde{B}$ until $\tilde{r} \perp \tilde{t}$, calling the new elements $\tilde{A}$ and $\tilde{\tilde{t}}$. 

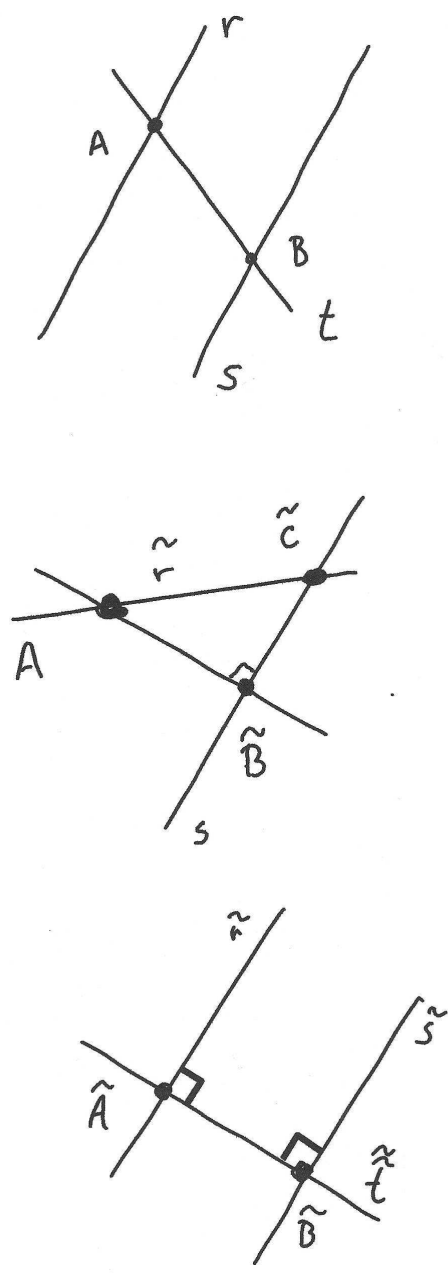

Figure 15. Another complicated case

Though the last two cases were rather complicated, we now have enough to summarize our results. First, let us define a pencil as the set of all lines parallel to one another or all lines intersecting in the same point. We can now say: three lines compose a pencil if and only if composing reflections of them yields a reflection in a line of the pencil. 


$$
\text { "Zeitler_Camp" — 2009/5/28 — 21:24 — page } 12 \text { — \#12 }
$$

It is quite natural to continue and ask about 4-fold reflections and beyond. Though we will stop the investigation here, we have really stumbled across all we need to handle higher order reflections because:

- $2 n$-fold reflections can be represented by 2 -fold reflections.

- $2 n+1$-fold reflections can be represented by 3 -fold reflections.

- Thus fundamental mappings of identity, $\sigma, \tau, \rho$ and $\sigma \tau$ are all of the possible compositions of reflections.

Now we are able to solve many complex geometric problems without any calculations, including those at the beginning of this article. We do not need any analytic geometry at all. What a wonderful insight - we have an entire algebra whose elements are reflections! As many of you may know, not only is this fun geometrically speaking, it has immediate practical applications in group theory. What a fascinating game mathematics can be!

\section{References}

[1] G. Ewald, Geometry: An Introduction, Belmont, 1971.

[2] T. J. Fletcher, Some Lessons in Mathematics, London, 1965.

[3] H. W. Guggenheimer, Plane Geometry and its Groups, San Francisco, 1967.

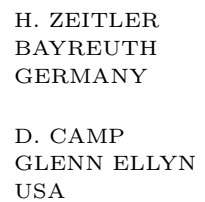

(Received May, 2007) 